\title{
Fat Bolus Impaction; a Rare Case Report
}

\author{
Dr.Santosh S Garag ${ }^{1}$, Dr. Shibani V Anchan ${ }^{2}$, Dr. Arun kumar.J.S ${ }^{3}$, \\ Dr. Santosh $\mathrm{MCB}^{4}$ \\ 1 \& 2 Assistant Professor Dept of ENT, 3 Professor Dept of ENT, \\ 4 Associate Professor Dept of Anaesthesiology \\ SDM college of Medical sciences \& hospital, Dharwad, Karnataka, India.
}

\begin{abstract}
An impacted esophageal foreign body is most often an urgent, rather than a life-threatening, medical situation. Typically, two types of foreign bodies are encountered: true foreign bodies (buttons, coins, pieces of balloon etc) and food-related foreign bodies. Ingestion of true foreign bodies generally occurs in persons less than 40 years old, with the vast majority being children. The incidence of true foreign body ingestion is also high in incarcerated individuals and in persons with psychiatric disorders. Food-related foreign bodies are more prevalent in persons who are over 60 years of age, who have esophageal disease, anatomic narrowing, motility disorders or who have recently consumed central nervous system depressants, especially ethanol. This article describes the clinical presentation, radiological findings and management of impacted mutton fat bolus as foreign body in an adult male patient.
\end{abstract}

Key Words; Foreign body, Adipose tissue, Endoscopy

\section{Introduction}

Swallowed and inhaled foreign bodies are a common problem and considered serious causes of morbidity and mortality [1]. Classically foreign bodies swallowed into esophagus present with dysphagia. An esophageal food bolus obstruction (or steakhouse syndrome) is a medical emergency caused by the obstruction of the esophagus by an ingested foreign body. It is usually associated with diseases that narrow the lumen of the esophagus, such as eosinophilic esophagitis, Schatzki rings, peptic strictures, webs, or cancers of the esophagus; rarely it can be seen in motility disorders of the esophagus, such as nutcracker esophagus. While some esophageal food boluses can pass by themselves or with the assistance of medications, some require the use of endoscopy to push the obstructing food into the stomach, or remove it from the esophagus. Depending on their nature, foreign bodies may either have uneventful gastrointestinal transitions or be impacted in a particular segment of the gut. Food bolus impaction is common in both children and adults. In children with recurrent food bolus impaction, one should rule out eosinophilic esophagitis. In adults an underlying stricture or narrowing of esophageal lumen due to growth should be kept in mind [1]. We present here a rare case of foreign body mutton fat bolus lodged in cricopharynx. Cases of mutton bones with meat bolus (muscle) have been reported. In an extensive review of literature we did not come across any such fat bolus impaction being reported.

\section{Case Report}

An adult male patient of age 37 yrs presented to the emergency department with a history of sudden onset of dysphagia and pain in the neck after having alcohol and meat meal. Patient was chronic alcoholic and habit of tobacco chewing. No previous history of any foreign body impaction. On examination oral cavity \& oropharynx was normal. Indirect laryngoscopy revealed pooling of saliva in both the pyriform sinus, with grayish mass like lesion behind the arytenoids. There was no respiratory distress. Neck examination revealed absence of laryngeal crepitus. Lateral and AP view x-ray of neck was taken, which revealed widening of soft tissue shadow opposite C5-C7 vertebrae with some radio-opaque shadow seen at C-6 level (Fig.1). Provisional diagnosis of mutton bone or mutton bone with underlying postcricoid growth was considered, as patient gave history of chronic alcoholism \& tobacco usage. Patient was posted for emergency direct laryngoscopy and removal of foreign body under GA. On direct rigid laryngoscopy there was grayish mass like lesion behind the arytenoids (Fig.2). Initially it was removed piecemeal with alligator forceps which did not bleed, hence foreign body was suspected and removed after deflating the cuff of endotracheal tube. It was a big fat bolus impacted at cricopharynx (Fig.3). After removal of the foreign body esophagoscopy was done to rule out any stricture or growth below the level of cricopharynx, esophagus was normal. Foreign body was sent for histopathology which confirmed that it was adipose tissue. Post operative period was uneventful. Repeat x-ray of the neck revealed persistent radio-opaque shadow that was calcified laryngeal cartilages which mimicked radio-opaque foreign body (Fig.4) 


\section{Discussion}

Foreign body impaction in the upper airway and digestive tract has been a problem since the earliest of reported history [1]. The foreign body spectrum was defined by Jackson as an object or substance foreign to the location where it is found. He classified them as exogenous and endogenous. An infinite variety of foreign bodies may be inhaled or swallowed. Groundnut, castor seed, pieces of brick, stones, earrings, pins and whistles are some of the common foreign bodies aspirated into the airway whilst fish bone, mutton piece, chicken bone and coins are the common foreign bodies swallowed into the food passage. Carelessness of parents (in case of children), poor vision, mental infirmity, drug addiction and rapid eating are some of the factors responsible for this. Dentures, excessive alcohol ingestion and intake of markedly cold fluids have also been incriminated in the literature [1]. The severity of the symptoms depends upon the site, size, composition and period for which the foreign body has been present. It can be life threatening thus needing prompt management. Fortunately, the vast majority of all swallowed objects pass through the gastrointestinal tract without a problem. Most upper GI foreign bodies are related to food impaction, with mutton or chicken bones most often found. Poor dentition, inadequate chewing, alcohol intoxication and eating while under sedation can precipitate this problem. If the foreign body lodges in the cricopharyngeal sphincter or esophagus, early endoscopic removal under general anesthesia is performed. Patients treated in this manner had no morbidity and a short hospital stay. Our case was unique in having mutton fat bolus stuck in cricopharynx leading to absolute dysphagia. Usually fat is soft and slippery doesn't get impacted, when cooked it becomes oily. The peculiar problem we faced with this foreign body was that since it was tightly impacted in cricopharynx and soft, thus initially removed piecemeal. Finally disimpacted by deflating the cuff of endotracheal tube and the foreign body was removed successfully. Lateral soft tissue neck X-ray is contributory in making the diagnosis. Widening of the prevertebral soft tissue is pathologic until proven otherwise as seen in our case. A simple but less precise rule is that on soft tissue plain X-rays, the pre-vertebral shadow should be less than one half the width of the corresponding vertebral body [2]. Eosinophilic esophagitis is a disease associated with dysphagia and has a seasonal variation in incidence [3]. There was a significant seasonal variation in the incidence of acute esophageal bolus impaction due to Eosinophilic esophagitis more during summer than winter [4]. Meat impacted in the proximal two thirds of the esophagus is unlikely to pass and should be removed as soon as possible. Meat impacted in the lower third frequently does pass spontaneously and the patient can safely wait, under medical observation, up to 12 hours before extraction [5]. Even if a meat bolus does pass spontaneously, endoscopy must be done later to assess (8090\%) underlying pathology. Additional modes of therapy include the use of sublingual nitroglycerin or nifedipine to relax the lower esophageal sphincter, but they are not usually as effective as intravenous glucagon [6]. A new technique using an endoscope which is modified such that the endoscope itself becomes a directvision suction device [7]. This technique was used successfully in seven patients with meat bolus impaction in the esophagus. The method proved quick and safe, and no complications occurred. Effervescent agents seem to be effective at resolving esophageal food obstruction but their use is not without risk of complications $[8,9]$. The ossification of laryngeal cartilage is part of the normal aging process. To date, ossification of the laryngeal cartilages has presented with foreign body-like impaction [10]. In the present case ossification of the laryngeal cartilages masqueraded as a foreign body. Albeit rare, clinicians and radiologists should be aware of this ossification which may mimic as a foreign body, widened prevertebral soft tissue may be an underlying pathology.

\section{Conclusion}

Foreign body at cricopharynx is most common presentation in otolaryngology practice. Routine conventional x-rays are useful before any intervention. Pre-vertebral widening with calcified laryngeal cartilages mimic radio-opaque foreign body in our case. But it was mutton fat (adipose tissue) bolus which widened the pre-vertebral region in radiographs. Absence of laryngeal crepitus made us to think underlying growth causing impaction of foreign body. However direct laryngoscopy and esophagoscopy confirmed the diagnosis.

\section{References}

[1]. Pavan Sardana, Arvind S Bais, V. P. Singh, Meeta Arora. Unusual Foreign Bodies Of The Aerodigestive Tract Indian Journal of Otolaryngology and Head and Neck Surgery Vol. 54 No. 2, April - June 2002125

[2]. C.A. Rojas, D. Vermess, J.C. Bertozzi, J. Whitlow, C. Guidi andC.R. Martinez. Normal Thickness and Appearance of the Prevertebral Soft Tissues on Multidetector CT. American Journal of Neuroradiology 30:136-141, January 2009

[3]. Hurtado CW, Furuta GT, Kramer RE. Etiology of esophageal food impactions in childrenJ Pediatr Gastroenterol Nutr. 2011 Jan; 52(1):43-6.

[4]. Larsson H, Bergquist H, Bove M. The incidence of esophageal bolus impaction: is there a seasonal variation? Otolaryngol Head Neck Surg. $2011 \mathrm{Feb} ; 144(2): 186-90$

[5]. Blair SR, Graeber GM, Cruzzavala JL, Gustafson RA, Hill RC, Warden HE, Murray GF: Current management of esophageal impactions. Chest 1993; 104:1205-1209.

[6]. Hin Hin Ko, Robert Enns, Review of food bolus management. Can J Gastroenterol. 2008 October; 22(10): 805-808.

[7]. Kozarek RA, Sanowski RA. Esophageal food impaction; description of a new method for bolus removal. Dig Dis Sci 1980; 25:100103. 
[8]. Jason Lee, Ross Anderson, Effervescent agents for oesophageal food bolus impaction

[9]. Rice B: Acute esophageal food impaction treated by gas-forming agents. Radiology 1983;146:299.

[10]. Na Rae Kim, Dong Young Kim, Hee Young Hwang et al: A case of ossified laryngeal cartilage mimicking a subglottic mass Received 1 June 2011 published online 16 September 2011. American Journal of Otolaryngology - Head and Neck Medicine and Surgery.

\section{Figure Legends}

Fig 1 Lateral view x-ray of neck showing prevertebral widening at C5-C7 with radiopaque shadow at C-6 level

Fig 2 Endoscopic picture showing foreign body at postcricoid area with pooling of saliva

Fig 3 Foreign body removed ( adipose tissue)

Fig 4 Lateral view x-ray of neck showing persistent radiopaque shadow at C-6 level after removal of foreign body

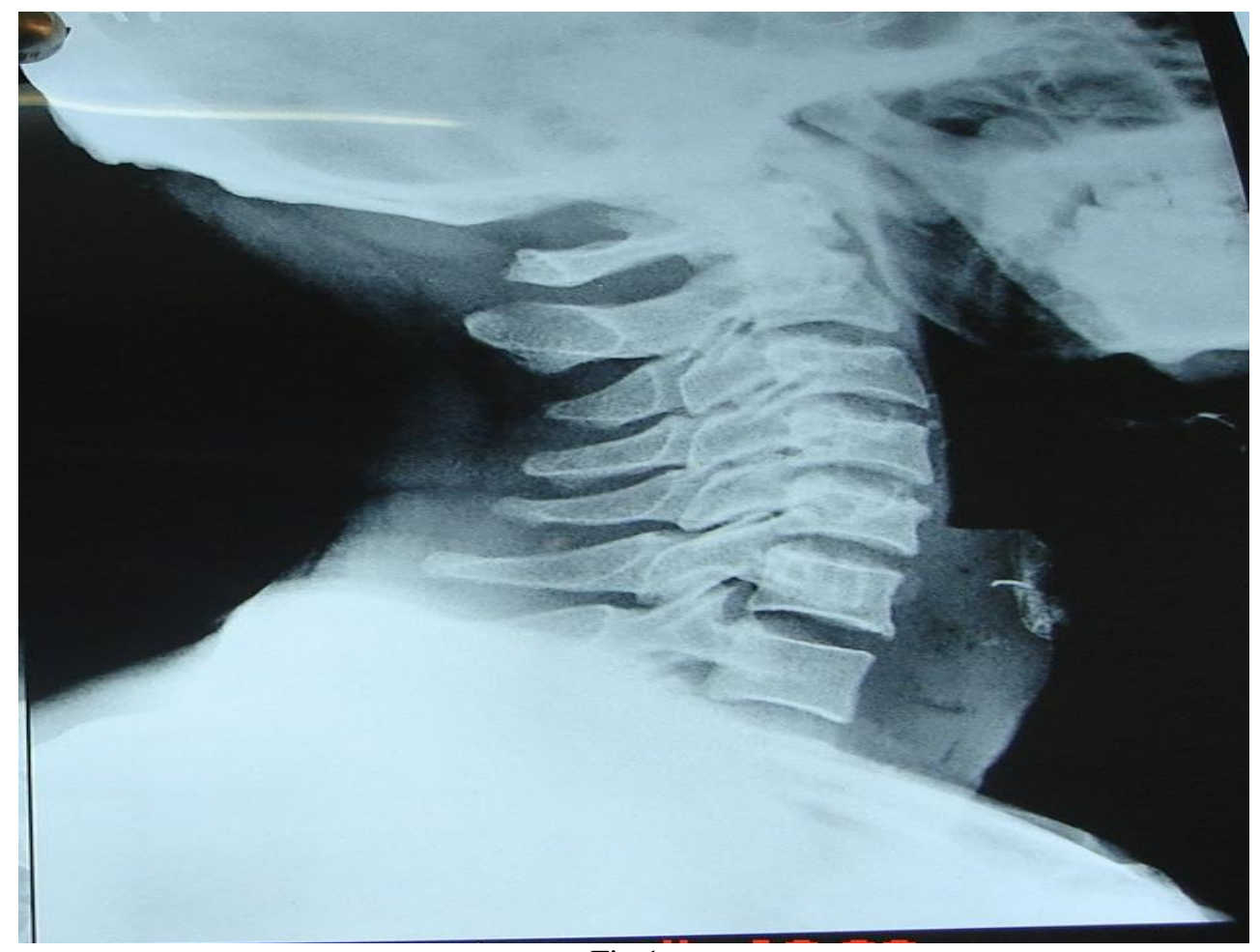

Fig 1

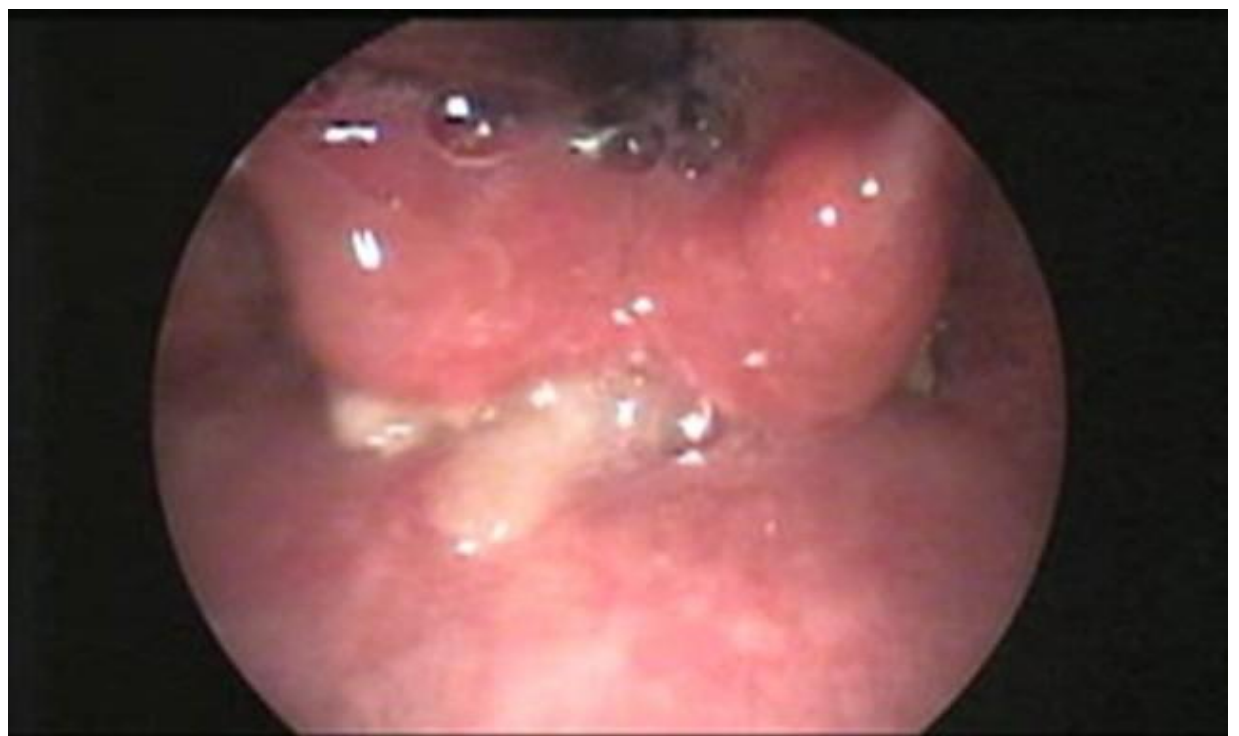

Fig 2 


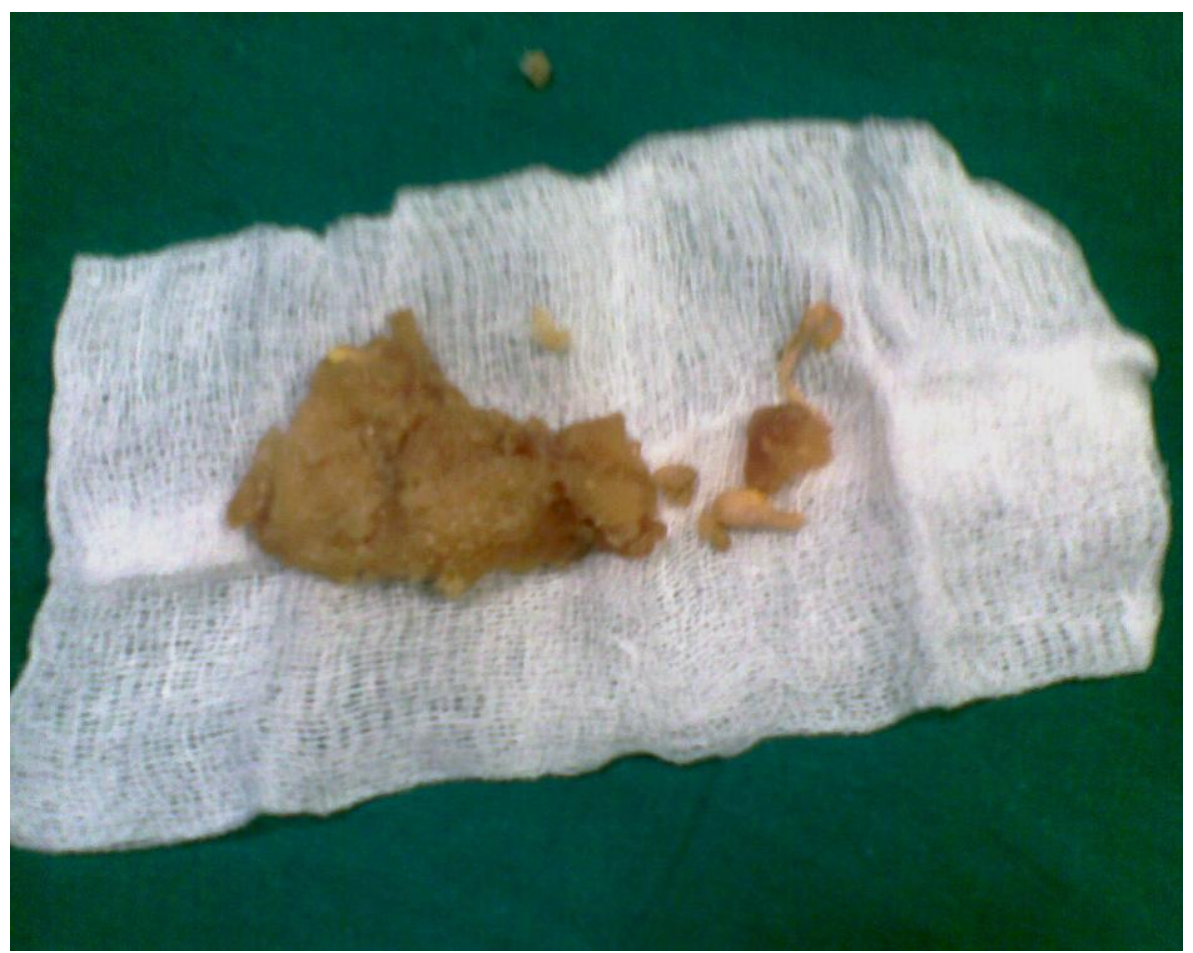

Fig 3

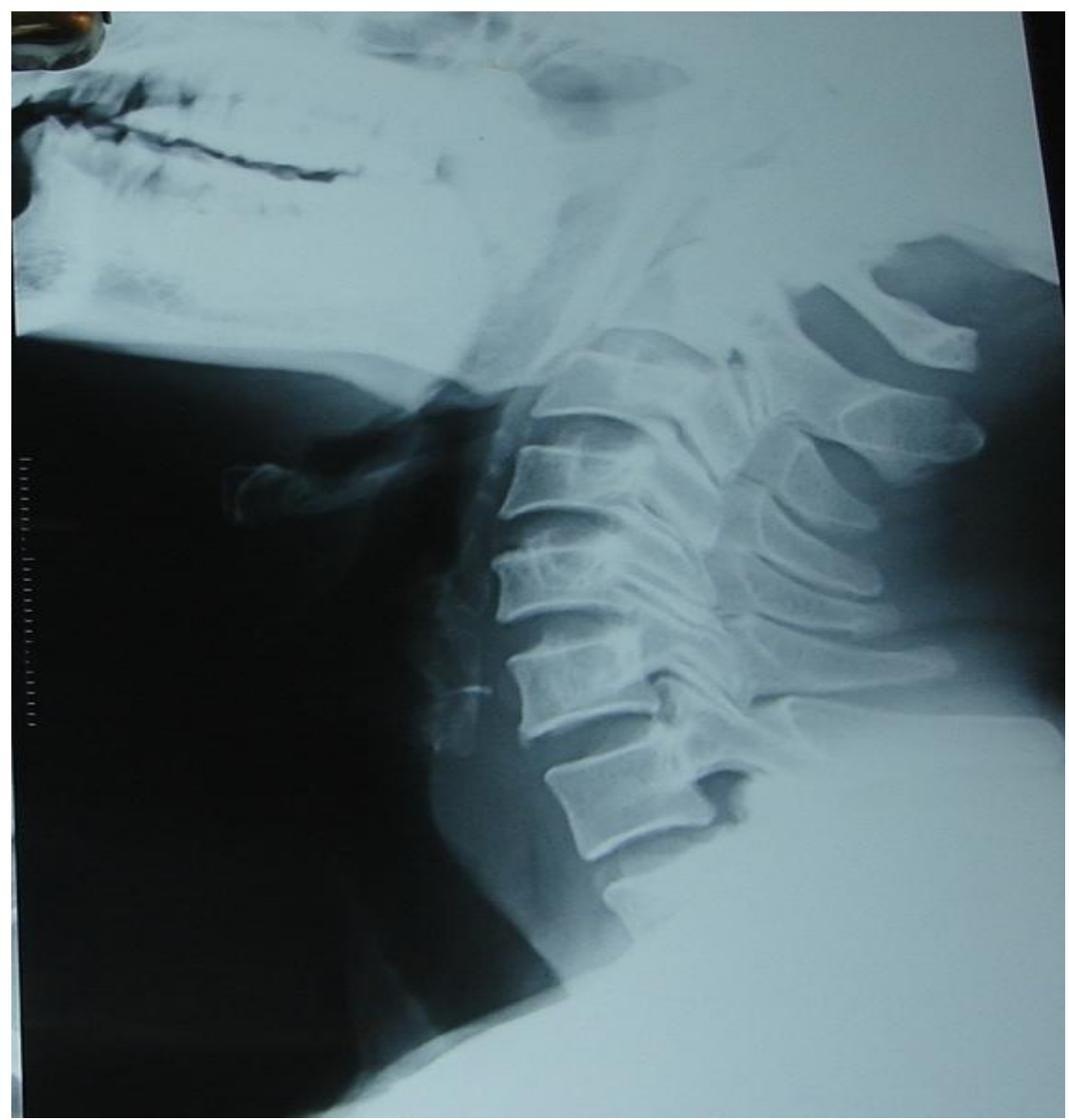

Fig 4 